\title{
Competencias administrativas en la calidad del servicio en las instituciones educativas del nivel inicial
}

Luis Alberto Núñez Lira ${ }^{11}$, Marisol Verónica Giordano Silva², Isabel Menacho Vargas ${ }^{3}$, Yrene Cecilia Uribe Hernández y Luis Rodríguez Fuentes ${ }^{5}$

\author{
Universidad Nacional Mayor de San Marcos, Lima, Perú ${ }^{1}$ \\ Universidad César Vallejo, Lima, Perú ${ }^{123}$ \\ Universidad San Ignacio de Loyola, Lima, Perú ${ }^{4}$ \\ Universidad de Granada, Granda, España ${ }^{5}$ \\ Orcid ID: https://orcid.org/0000-0003-3542-9117 \\ Orcid ID: https://orcid.org/0000-0001-6111-7016 \\ Orcid ID: https://orcid.org/0000-0001-6246-4618 \\ Orcid ID: https://orcid.org/0000-0001-5893-9262
}

Recibido: 12 de marzo de 2018

Aceptado: 07 de noviembre 2018

\section{Resumen}

Se buscó determinar la influencia de las competencias administrativas desarrolladas en las directoras sobre la calidad del servicio educativo que ofrecen las instituciones educativas del nivel inicial en la Unidad de Gestión Local 07, Perú. Esta investigación se enmarca dentro del modelo positivista y de tipo básica-sustantiva, con nivel explicativo y de diseño transversal y no experimental. Fueron 155 docentes del nivel inicial que conformaron una muestra probabilística, aleatoria simple; se aplicaron dos escalas de opinión o percepción como instrumentos para la recolección de datos cuya validez y confiabilidad fueron establecidas previamente; los datos fueron procesados estadísticamente mediante el análisis de regresión. Se halló que el 71,3\% de las profesoras de inicial percibían que en las directoras de las Instituciones Educativas de nivel Inicial del distrito habían desarrollado las competencias administrativas y que el $85 \%$ de las profesoras de inicial perciben que el servicio que brindan en su escuela es de alta calidad y para el $12,5 \%$ el servicio es de calidad, concluyéndose que la influencia de las capacidades administrativas de las directoras fue del 45,63\% y sobre la percepción de alta calidad en el servicio que se brinda en las instituciones educativas del nivel inicial de la UGEL 07.

Palabras clave: Gestión educativa, capacidades administrativas, calidad de servicio

\section{Administrative competences in the quality of service in educational institutions of}




\title{
the initial level
}

\begin{abstract}
We sought to determine the influence of the administrative competences developed by the directors on the quality of the educational service offered by the educational institutions of the initial level in the UGEL 7. This study is within the positivist model and is basic-substantive with an explanatory level and a transversal and nonexperimental design. There were 155 teachers from the initial level who made up a random, simple probabilistic sample. Two scales of opinion or perception were applied as instruments for data collection whose validity and reliability were previously established; the data were processed statistically by regression analysis. It was found that $71.3 \%$ of the initial teachers perceived that administrative competences had been developed in the directors of the district IEIs and that $85 \%$ of the initial teachers perceive that the service they provide in their school is of high quality and for $12.5 \%$ the service is of quality, concluding that the influence of the administrative abilities of the directors was $45.63 \%$ and on the perception of high quality in the service provided in the educational institutions of the initial level of the UGEL 07.
\end{abstract}

Keywords: Educational management, administrative capacities, quality of service. 


\section{Introducción}

Desde una mirada en el contexto educativos peruano y considerando los datos estadísticos disponibles se observa que la asistencia de niños y niñas a las Instituciones Educativas de nivel Inicial [IEI] desde el 2001 y hasta el 2012 se ha incrementado significativamente de $53 \%$ a $75 \%$. Un aumento que sigue siendo desigual en la zona urbana en relación con la rural, del $59 \%$ al $78 \%$ en el primer caso y del $44 \%$ al $66 \%$ en la restante, si bien se aprecia un aligera una reducción de la brecha en torno al 3\%. No obstante, los recursos recibidos siguen siendo insuficientes, en 2017 la representación parlamentaria peruana aprobó la Ley de Presupuesto Público en la que se asignó el 10,85\% al nivel educativo inicial, equiparándose con países como México y Guatemala pero aún distante de otros como España (14\%) y que Chile (16\%) a pesar de que funcionarios del Instituto Peruano de Economía (IPE) reconocieron que los retornos sociales más altos en la educación se encuentran en el nivel inicial, donde que cada sol que se invierta en inicial tiene un retorno social de más de diez.

Sin embargo, deberá tenerse en cuenta que no por ser mayor la cobertura económica el impacto en el rendimiento del niño en la escuela aumentaría, pues la calidad con la que se ofrece la educación también depende en gran medida de su modelo de gestión y la metodología de su enseñanza. Es evidente que se deben perfeccionar las capacidades de gerencia directiva y pedagógica en los establecimientos como forma de optimizar los recursos y talento humano del que disponen (Del Arco, 2007).

Por otro lado, y a pesar del convencimiento de que una educación inicial de calidad favorece tanto las oportunidades de aprender como el desarrollo socioemocional, cognoscitivo y psicomotor de los estudiantes de inicial, en la mayoría de las instituciones los servicios educativos siguen sin brindar cuidados eficaces, ni se propician entornos educativos debidamente implementados, cálidos y motivadores.

En los últimos años, se ha obviado que los niños desarrollan sus capacidades de aprendizaje atendiendo al estado de madurez en el que se encuentran. Asimismo, el acompañamiento que se les provee no respeta en muchos casos su ritmo para aprender, sobre exigiendo al niño o a la niña, como cuando por complacer a los padres o por querer demostrar una falsa eficiencia se pretende que escriban cuando aún se hallan en transcurso de desarrollo motriz fino.

Otro aspecto que en gran medida resulta deficiente es que el trabajo pedagógico no aborda el desarrollo integral que se da en los niños y las niñas, considerando desde 
el desarrollo del movimiento, las emociones, la comunicación (verbal y no verbal) hasta llegar al pensamiento.

A todo ello se agrega las condiciones en la que los estudiantes deben ser atendidos en las instituciones de gestión pública, muchas de ellas con una infraestructura física descuidada y deterioro de sus servicios básicos; con mobiliarios vetustos e inadecuados para la formación de niños, con insuficientes materiales proveídos por el Minedu para la población que se atiende y espacios poco adecuados, lo que representa un condicionante en el desarrollo del currículo (Rodríguez, 2014).

Posiblemente, en un recorrido por las IEI la reseña de deficiencias presentada se iría incrementando, siendo más desalentador por el convencimiento de que ello no cambiará en el corto plazo, ante lo que surge la pregunta ¿qué hacer en estas condiciones? La respuesta estaría en la actuación de la persona que dirige la institución, es decir de las directoras, mayormente docentes del nivel inicial. Y si nuevamente se dirige la mirada a la situación de las IEI, se verán diferencias entre ellas, siendo todas estatales y recibiendo el mismo trato del estado. Probablemente esto se deba a las diferencias en sus capacidades administrativas para gestionar los exiguos recursos que poseen.

Por ello se decidió estudiar un grupo de instituciones educativas del nivel inicial de gestión estatal. En tal sentido, las instituciones educativas del nivel inicial se establecieron como las unidades de análisis, siendo las docentes las llamadas a evaluar las capacidades en sus directoras y el servicio que se brinda a los estudiantes. Así también, y con la finalidad de limitar en lo posible la influencia de variables intervinientes, la selección de IEI se realizó sobre un mismo sector con condiciones socioeducativas y socioeconómicas similares.

Sin que ello signifique dejar de exigir al estado y a la sociedad en su conjunto las mejoras necesarias, se consideró prioritario atender desde la mejor manera posible a los niños y niñas de educación inicial por cuanto representan las generaciones futuras y que no hacerlo se les estaría condenando a ser a los grupos más rezagados en una sociedad que pretende ser igualitaria. En tal sentido, es necesario pasar de la suposición de que estas capacidades administrativas marcan la diferencia a su confirmación o denegación; de allí que en esta investigación se pretendió determinar si su grado de influencia es suficiente argumento para el diseño de estrategias destinadas a desarrollar las capacidades que una directora requiere para administrar exitosamente los recursos de una IEI en pro de brindar un servicio de calidad en este nivel. 
Diversos investigadores afirman que es viable lograr la mejora de la calidad en el sector siempre y cuando exista una verdadera autonomía para poder cambiar la mentalidad y paradigma pedagógico y darle orientación lógica al sistema social a partir del sistema educativo. A esto hay que agregar la correlación "pertinencia-relevancia-equidad-protección" de los derechos del niño y humano (Cossio 2014, Beresaluce 2008, Blanco 2005).

\section{Competencia administrativa}

Son el conjunto de capacidades desarrolladas por los trabajadores que les permite realizar un conjunto de actividades - diagnosticar-organizar-tomar decisionesarticulando con los recursos y talento humano disponible que posee la organización para satisfacer a los usuarios (Ayala 2005, Chiavenato 2005), apreciándose el esfuerzo para el funcionamiento de cualquier organización.

Desde el punto de vista sistémico, es un modelo de gestión que permite al gestor la evaluación de un conjunto de capacidades específicas (conocimientos-habilidadesactitudes) en el puesto de trabajo (Alles 2016).

Estas competencias administrativas de los docentes-gestores encargados de las instituciones educativas son evaluables en la medida en que logren estandarizarse en el nivel. Situación compleja en tanto que las instituciones formadoras no han logrado establecer una misión visión compartida, cuyos enfoques administrativos y pedagógicos no necesariamente están alineados al ente rector.

Desde esta perspectiva se debe plantear para los gestores el desarrollo integral que considere el aprendizaje reflexivo orientado a tomar decisiones para resolver los problemas educacionales y resolver el tema de la evaluación de las competencias del gestor. En la praxis se considera mayormente el desarrollo de las estrategias metodológicas, -como competencias docentes- y las administrativas como el cúmulo de documentos que justifiquen su actividad pedagógica. Al igual que en México, la docencia gira en torno al modelo educativo (constructivistas) del Diseño Curricular establecido por normas más que por el desarrollo de actividades de gestión administrativa institucional (Castro 2015).

Así, el proceso de profesionalización docente se enfocó al desarrollo de competencias, la mayoría de ellas técnicas y tecnológicas, que solo buscan la implementación del currículo de acuerdo con los designios del ente rector. Desde los años 90 se implementaron estos procesos a través de la llamada formación continua en servicio, donde los directivos y docentes resuelven situaciones concretas haciéndolos 
“eficientes para el sistema” (Bolivar, 2009).

De esta manera, la formación en servicio ha buscado desarrollar diversas habilidades -planificación-metodología-evaluación- poniendo el énfasis en lo instrumental para poder evaluar y estandarizar el desempeño del docente.

Sin embargo, se debe considerar que la mejora de competencias de los responsables de la gestión educativa tiene como fin optimizar sus funciones de gestión, centrándolas en el logro de los objetivos misionales, perfeccionar el desempeño, elevar el conocimiento de sus funciones y alinear la visión, forjando verdaderos líderes y mejorando la calidad del servicio brindado (Mejía-Giraldo, Bravo-Castillo y MontoyaSerrano 2013).

Desde esta perspectiva, el enfoque por el desarrollo de competitividades orienta hacia la calidad en la praxis docente, generando la dependencia del desempeño en la calidad. Por ello, mirada la competencia como generadora de valor a través del desarrollo cognitivo, la aptitud y sapiencias técnicas se convertirá en potencial para la institución educativa aunada al compromiso y lealtad (Medina, Armenteros, Guerrero y Barquero 2012).

Por tanto, ser gestor en una institución educativa requiere el desarrollo de las competencias que permitan consolidar la eficiencia en logro de metas y objetivos propuestos, lo que implica una formación permanente (Díaz, Núñez y Cáceres 2018).

Se han considerado los siguientes factores de las competencias administrativas para esta investigación:

- Planificación: es el proceso de visualizar prospectivamente los resultados y metas sobre las acciones y actividades del plan cronogramados, presentando objetivos institucionales y procedimientos establecidos, tratando de asegurar que ocurra. (Salazar y Romero 2006). Por ello, saber planificar no solo depende del dominio cognitivo, sino también del dominio técnico y actitud positiva de liderazgo para la toma de decisiones permitentes (Núñez, Bravo, Cruz e Hinostroza 2018).

- Organización: es el proceso para ordenar, distribuir el trabajo y los recursos entre la comunidad educativa, que permitan lograr las metas organizacionales. Lograr la unidad entre los miembros, con relaciones sociales estables, hace viable la organización institucional, en un proceso de evolución constante (Escudero, Delfín y Arano 2014).

- Ejecución: operar eficientemente los diversos sistemas de personal, presupuesto, 
contabilidad y control adecuados a la institución educativa, aplican do los diversos dispositivos y normas técnicas y procedimientos de la infraestructura y equipamiento educativo. Esto nos lleva a la motivación, dirección de las actividades, estableciendo canales de motivación (Majad 2016).

- Control: es la función que permite asegurar el logro de metas y objetivos establecidos en el plan, de manera que permiten que las actividades planificadas se realicen de acuerdo al contexto social (Dextre y Del Pozo 2012).

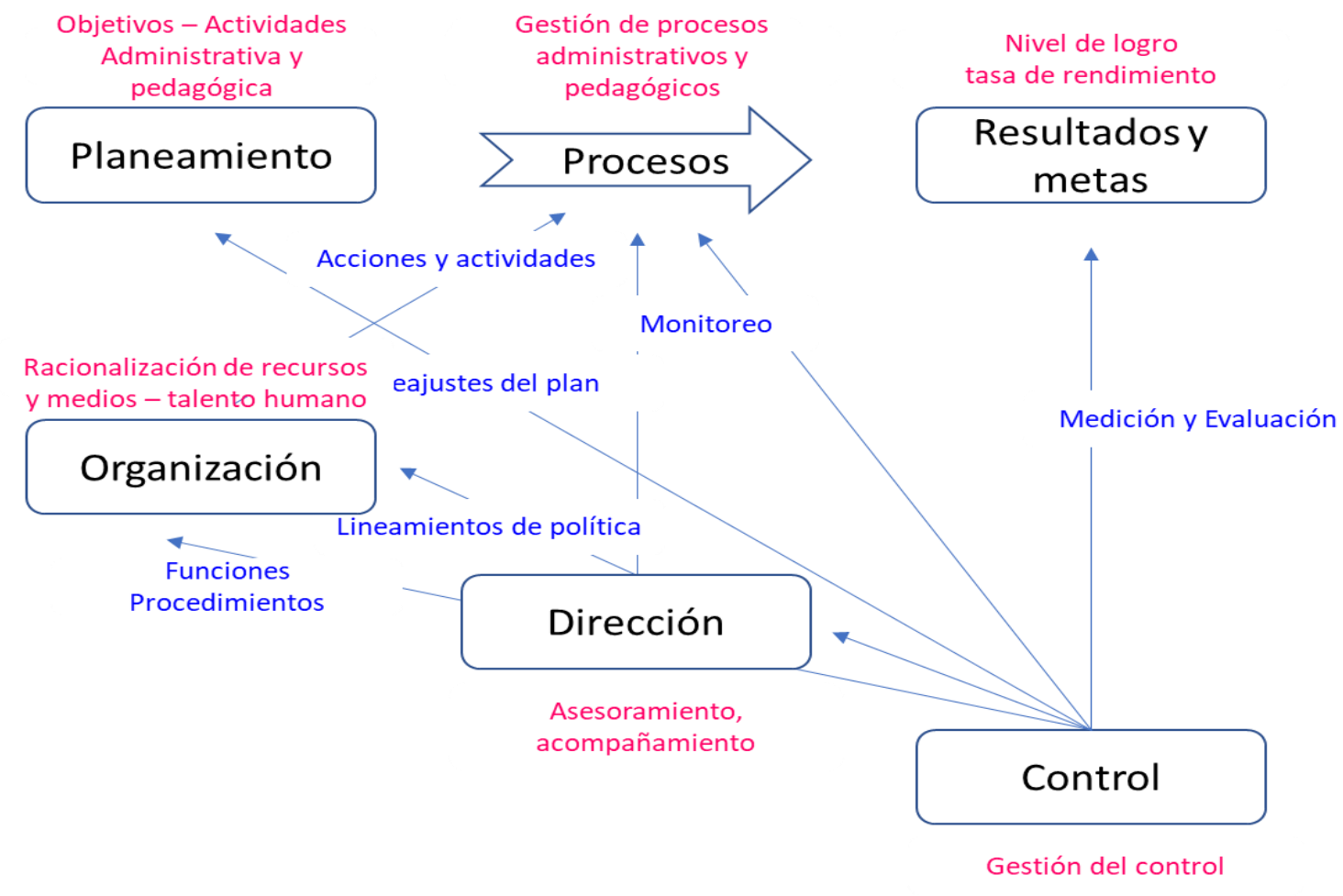

Figura 1. La gestión administrativa: una mirada funcional y por proceso

\section{Calidad del servicio educativo}

La calidad del servicio educativo, desde el punto de vista legal -Constitución Política del Perú (1993); la Ley General de Educación $N^{\circ} 28044$, la Ley del Sistema Nacional de Evaluación, Acreditación y Certificación de la Calidad Educativa (Ley SINEACE, 28740) y el Decreto Supremo $N^{\circ} 018$ - 2007 - ED-, es considerada como reto que se debe alcanzar el máximo nivel en la formación orientada al desarrollo humano, la formación de ciudadanos y el aprendizaje para la vida.

Para la Organización para la Cooperación y el Desarrollo Económicos [OCDE], 2001 la educación es de calidad cuando los estudiantes adquieren conocimientos- 
habilidades-destrezas-actitudes para su desarrollo como persona adulta (Miranda 2011) y la inclusión del alumnado (Gallego y Rodríguez, 2014).

Para Miranda (2011), los diversos actores educativos tienen su propia versión de la definición de calidad, debido a su mirada del contexto educativo. Esta realidad implica la necesidad de tener mesas de diálogo con la comunidad educativa con la finalidad de concertar metas y objetivos (Lorenzo, 2012).

Pero hablar de calidad va ligado a evaluación y a estándares (lo ideal), superando la visión subjetiva -percepción- cuando el usuario ve satisfecho sus necesidades, sea como producto sea como servicio (Cano 1998). Por ello, el establecimiento de indicadores de evaluación como producto de la perspectiva teórica que respaldan el análisis e interpretación de esa realidad.

Sin embargo, es cuestionable, en la medida que un sistema de evaluación de la calidad educativa solo permitirá legitimarse en su funcionamiento (normalización) pero no necesariamente si el sistema educativo es el esperado por la sociedad, para dar respuesta coherente a la problemática educativa y social.

En la línea trazada, la evaluación del servicio educativo serviría para legitimar un sistema, pero al menos habría que exigirle eficiencia de la gestión con la finalidad de lograr los objetivos propuestos en la institución educativa inicial.

Asimismo, desde la perspectiva de los padres de familia y estudiantes su requerimiento está en función de satisfacer sus expectativas y motivación conducentes a coadyuvar la calidad educativa ligada a la legitimación del sistema tratando de compatibilizar las dos miradas expresadas.

Para la United Nations Educational, Scientific and Cultural Organization [UNESCO], 2007, la calidad educativa está conformada por los siguientes factores o dimensiones:

- Relevancia: referida al atributo y finalidad de la educación. También referido a valorizar en el marco de la Ley de Educación.

- Pertinencia: referida a la conveniencia y oportunidad de la educación. Desde esta perspectiva, los indicadores clase para este factor son "respeto, diversidad y participación, flexibilidad y mecanismo de adaptabilidad".

- Equidad: referida a la justicia y equidad. Busca la igualdad de oportunidades por sexo, nivel de ingreso, condiciones sociales, económicas y étnicas.

- Eficacia: referida al logro de los objetivos educacionales planteados. Para ello es 
clave lograr el acceso y conclusión a la educación y logros académicos.

- Eficiencia: referido al máximo de resultados con menor esfuerzo, sea de tiempo y energía.

A partir de lo anterior se planteó como objetivo determinar la influencia de las capacidades administrativas de las directoras sobre la calidad del servicio que se brinda en las instituciones educativas del nivel inicial de la UGEL 07.

\section{Materiales y métodos}

La investigación desarrollada correspondió a un diseño no experimental, donde no hubo manipulación de variables, y transversal porque los datos recogidos fueron recolectados en un solo momento. Asimismo, se desarrolló una investigación básica sustantiva, porque lo que busca es el incremento de conocimientos científicos (Hernández, Fernández y Baptista 2014; Murillo 2011; Galán 2010; Vera 2008; Zorrilla 1993).

La población estaba conformada por 338 profesoras del nivel inicial que enseñaban en las instituciones educativas del nivel y que pertenecían a la jurisdicción de la UGEL 07. El tamaño de la muestra de estudio fue de 155 profesoras cuyo muestreo se estableció aleatoriamente. Los instrumentos utilizados fueron las encuestas donde a través de preguntas a la población permite recoger información sobre la variable de investigación (Paladines 2012).

El procesamiento de la información recogida se ha realizado a través de la estadística descriptiva de las variables y la contrastación de las hipótesis a través de la regresión logística multinomial.

\section{Resultados}

\section{Análisis descriptivo}

La información recogida ha permitido establecer en la tabla 1 que el 71,3\% de las profesoras del nivel inicial han percibido que en las directoras de las IEI del distrito habían desarrollado las competencias administrativas, lo que les permitiría ejercer adecuadamente su gestión educativa. Sólo el 28,7\% tenían las competencias poco desarrolladas 
IEI de Chorrillos, UGEL 07, 2018

Niveles

Poco desarrolladas

Desarrolladas

Fuente propia
Frecuencia relativa

$28,7 \%$

$71,3 \%$

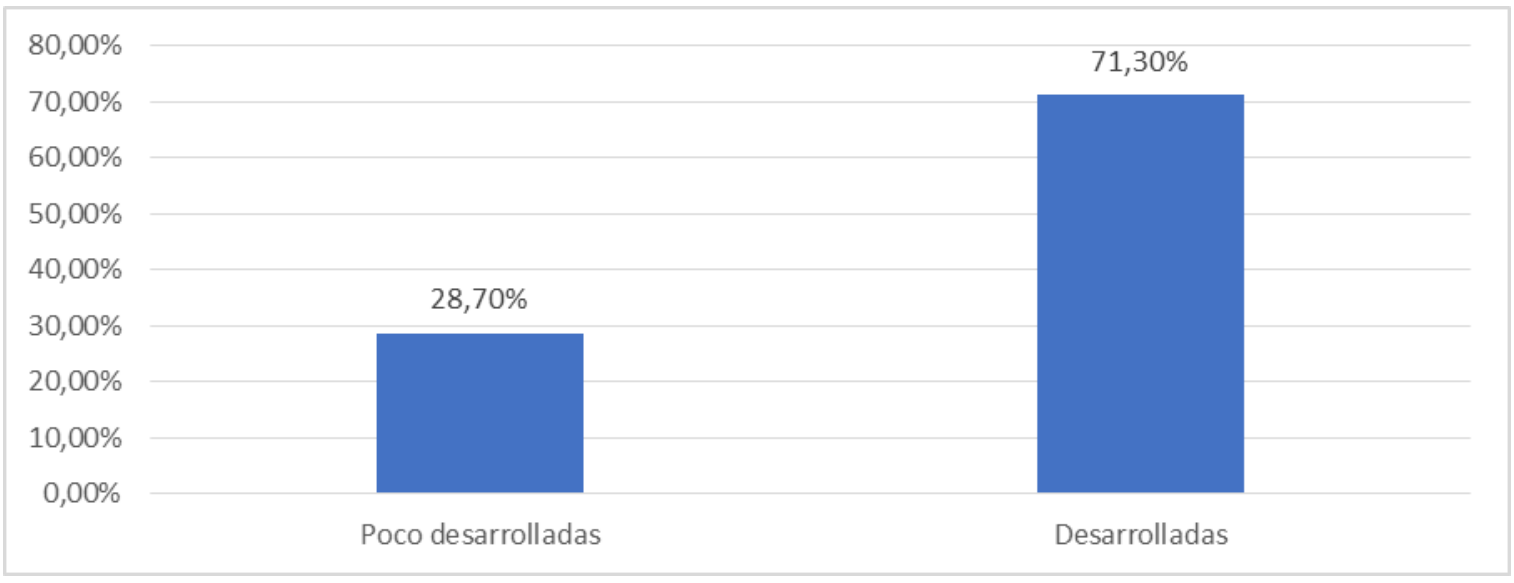

Figura 1. Percepción docente del desarrollo de competencias administrativas

Estos resultados, referidos en la tabla 2 sobre las competencias específicas desarrolladas, en el promedio el $70 \%$ de ellas consideraron que el mayor desarrollo alcanzado fue de la planificación y la organización de los procesos de gestión; el $52,50 \%$ consideró que el control se había desarrollado en las directoras; y el 46,30\% que había desarrollado poco, se ubicó pues a este aspecto estudiado como el de menor desarrollo en las competencias administrativas de las directoras, más aun si se tiene un cuenta que es el único aspecto en que el 1,3\% de las participantes consideraron que no se había desarrollado en las directoras.

Tabla 02

Percepción docente del desarrollo de competencias administrativas en directoras por dimensiones de las IEI de Chorrillos, UGEL 07, 2018

\begin{tabular}{lcccc}
\hline Dimensiones & Planificación & Organización & Dirección & Control \\
\hline No desarrolladas & $0,0 \%$ & $0,0 \%$ & $0,0 \%$ & $1,3 \%$ \\
Poco desarrolladas & $30,0 \%$ & $28,7 \%$ & $40,0 \%$ & $46,3 \%$ \\
Desarrolladas & $70,0 \%$ & $71,3 \%$ & $60,0 \%$ & $52,5 \%$ \\
Total & $100,0 \%$ & $100,0 \%$ & $100,0 \%$ & $100,0 \%$ \\
\hline
\end{tabular}

Fuente propia 


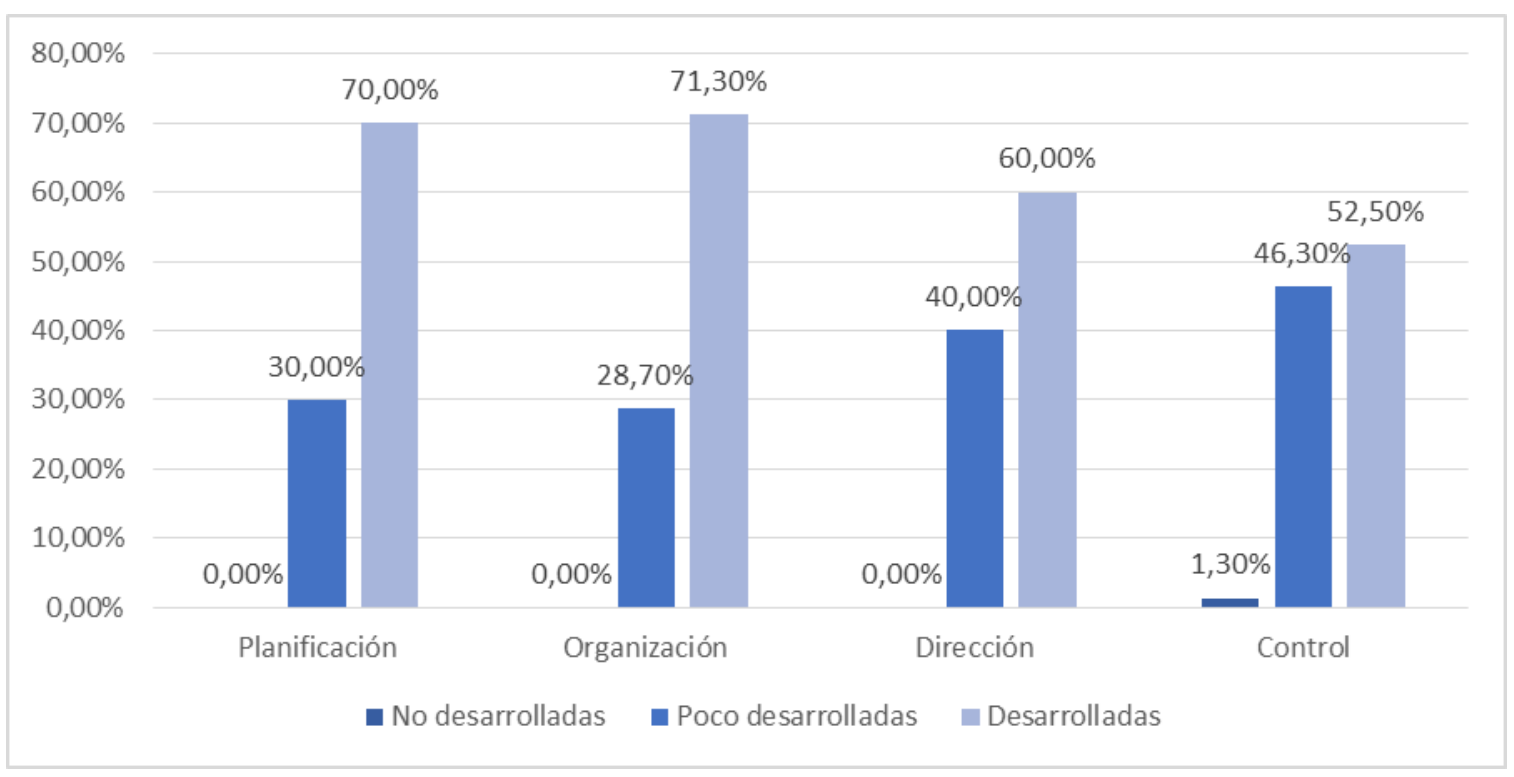

Figura 2. Percepción docente del desarrollo de competencias administrativas por dimensiones.

De los datos que se observan en la tabla 3 se logró establecer que el $85 \%$ de las profesoras de inicial perciben que el servicio que brindan en su escuela es de alta calidad. El 12,5\% percibe que es de calidad media y el 2,5\% percibe que el servicio es de baja calidad.

Tabla 03

Percepción de la calidad del servicio que se brinda en las IEI de Chorrillos, UGEL 07, 2018

\begin{tabular}{lc}
\hline Niveles & Frecuencia relativa \\
\hline Servicio de baja calidad & $2,5 \%$ \\
Servicio de calidad & $12,5 \%$ \\
Servicio de alta calidad & $85,0 \%$ \\
Total & $100,0 \%$ \\
\hline
\end{tabular}

Fuente propia 


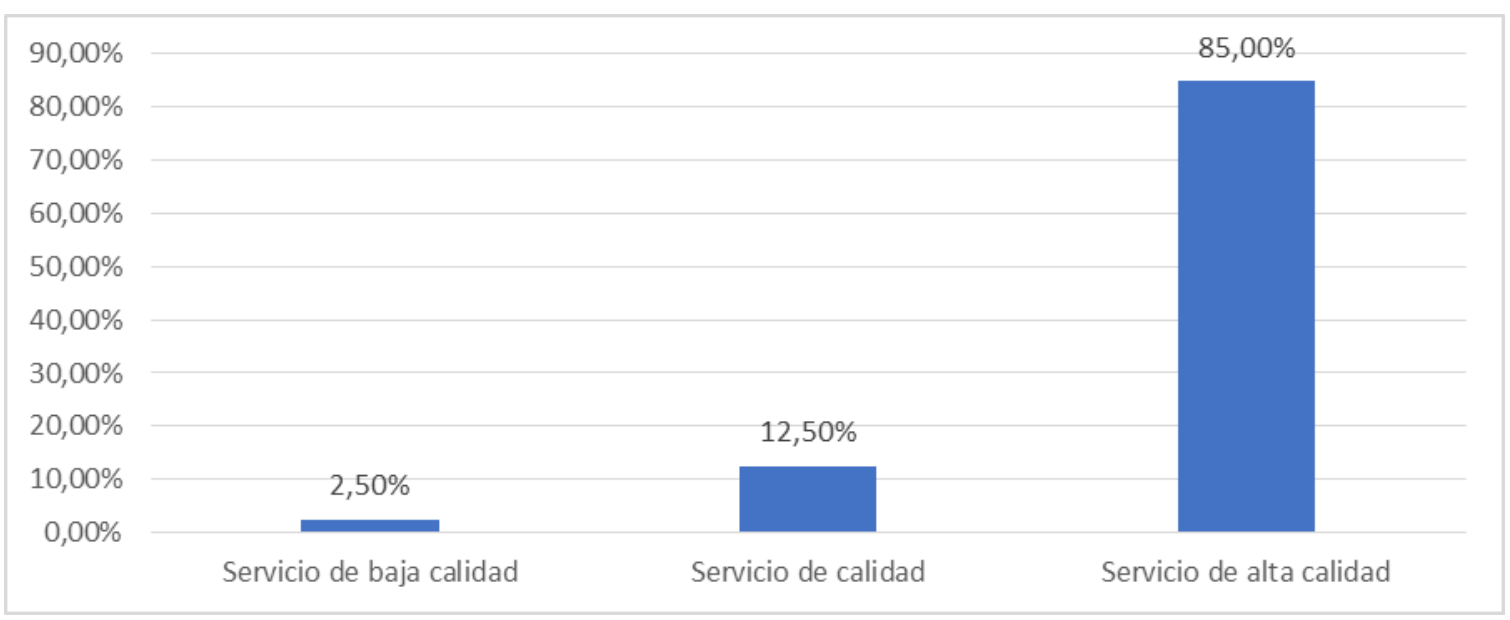

Figura 3. Percepción de la calidad del servicio

De los datos que se observan en la tabla 4, se logró establecer que el 85\% de las profesoras de inicial perciben que el servicio que brindan en su escuela es de alta calidad y para el 12,5\% el servicio es de calidad aceptable. De forma global, según el 97,5\% de estas percepciones en las instituciones educativas del nivel inicial, brindan un servicio educativo de calidad.

Tabla 04

Percepción de la calidad del servicio por dimensiones que se brinda en las IEI de Chorrillos, UGEL 07, 2018

\begin{tabular}{lccrrc}
\hline Niveles & Pertinencia & Relevancia & Equidad & Eficacia & Eficiencia \\
\hline De baja calidad & $1,3 \%$ & $1,3 \%$ & $0,0 \%$ & $2,5 \%$ & $3,8 \%$ \\
De calidad & $32,5 \%$ & $23,8 \%$ & $12,5 \%$ & $21,3 \%$ & $36,3 \%$ \\
De alta calidad & $66,3 \%$ & $75,0 \%$ & $87,5 \%$ & $76,3 \%$ & $60,0 \%$ \\
Total & $100,0 \%$ & $100,0 \%$ & $100,0 \%$ & $100,0 \%$ & $100,0 \%$ \\
\hline
\end{tabular}

Fuente propia 


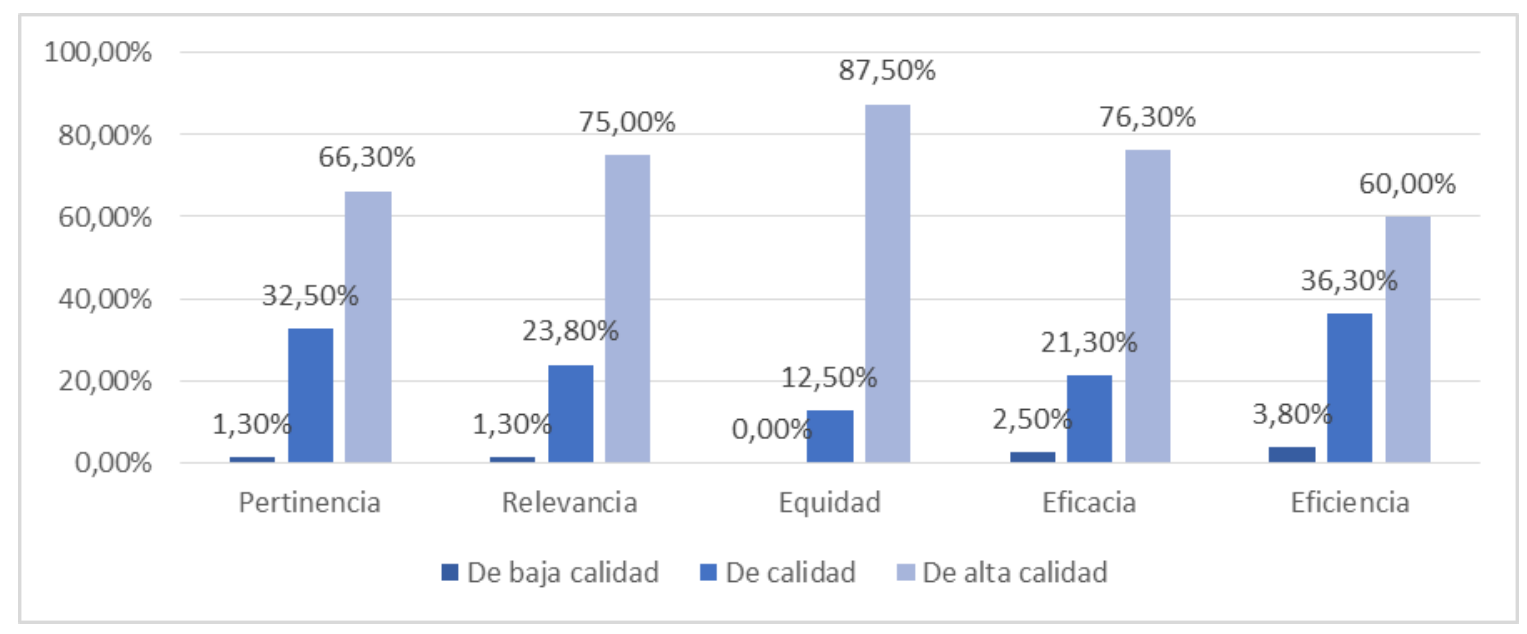

Figura 4. Percepción de la calidad del servicio por dimensiones

Los resultados de la contrastación de la hipótesis general, presentados en la tabla 5 , indica que la razón de verosimilitud del chi-cuadrado (LR chi2) presentó un valor de 35,79 correspondiéndole un p valor (Prob > chi2) de 0,000, lo que indicó que el modelo encajaba mejor que un modelo sin predictores. Asimismo, el valor del pseudo $\mathrm{R}$ cuadrado (R2) de 0,4563 indicó que el 45,63\% de la variabilidad de la calidad de servicio es explicada por las competencias administrativas. Por otro lado, para el servicio de baja calidad y el servicio de calidad, el $\mathrm{p}$ valor de $\mathrm{Z}(\mathrm{P}>|\mathrm{z}|)$ para las competencias administrativas es mayor que 0,05 , luego no resultó significativo.

Esto nos indica que el modelo es adecuado, con un 45,63\% de explicación de la variabilidad de la calidad de servicio por el desarrollo de las competencias administrativas. Al no existir evidencias estadísticas de que hay influencia en las percepciones de baja calidad y calidad aceptable en el servicio se infiere que la influencia sería sobre la percepción de alta calidad, aceptándose la hipótesis de que las capacidades administrativas de las directoras influyen positivamente sobre la calidad del servicio que se brinda en las instituciones del nivel inicial.

Tabla 5

Resultados del análisis de regresión logística multinomial de la calidad de servicio y competencias administrativas

\begin{tabular}{|c|c|c|}
\hline Regresión logística multinomial & Número de observaciones & $=80$ \\
\hline & LR chi2(2) & $=35,79$ \\
\hline & Prob. > chi2 & $=\mathbf{0 , 0 0 0 0}$ \\
\hline & Pseudo R2 & $=\mathbf{0 , 4 5 6 3}$ \\
\hline
\end{tabular}




\begin{tabular}{|c|c|c|c|c|c|c|}
\hline \multirow{4}{*}{$\begin{array}{l}\text { De baja } \\
\text { calidad }\end{array}$} & \multicolumn{6}{|l|}{ Competencias } \\
\hline & Administrativas & $-19,089$ & 4337,988 & $-0,00$ & 0,996 & $-8521,389 ; 8483,212$ \\
\hline & \multicolumn{6}{|l|}{ Desarrolladas } \\
\hline & Constante & 1,7048 & 0,7687139 & $-2,22$ & 0,027 & $-3,21146 ;-0,19816$ \\
\hline & Competencias & & & & & \\
\hline \multirow[t]{3}{*}{ De calidad } & Administrativas & $-19,089$ & 1940,007 & $-0,01$ & 0,992 & $-3821,433 ; 3783,256$ \\
\hline & Desarrolladas & & & & & \\
\hline & Constante & $-0,0954$ & 4369315 & $-0,22$ & 0,827 & $-0,951743 ; 0,760997$ \\
\hline
\end{tabular}

Fuente: Stata 14.1

\section{Discusión}

Los hallazgos estadísticos nos indican que la calidad del servicio es explicada por las competencias administrativas que presentan las directoras de las instituciones educativas del nivel inicial, en el 45,63\% de los informantes. Y esto confirma que la calidad está correlacionada con los procesos de evaluación, sea visto a través de procesos propiamente evaluativos sea también de la percepción de empoderamiento e implicación de los usuarios, internos o externos a la organización, tal y como también apuntara Contreras (2016).

Beresaluce (2008) en su investigación explicaba que la calidad del servicio educativo va a depender de la capacidad directiva de trabajar con creatividad y autonomía, situación muy limitada en la realidad peruana, cuya gestión se ha convertido en operativa al estar bajo la rigidez de los dictados del Ministerio de Educación.

Para Chiavenato (2005) es fundamental que entre las competencias administrativas de un director o gestor se encuentren las capacidades de crear, diseñar y mantener para alcanzar con eficiencia metas establecidas. Por ello, las funciones administrativas de planeación, organización, integración de personal, dirección y control son clave para alcanzar la calidad.

Como puede apreciarse, el esfuerzo humano -tanto de directivos como de la comunidad educativa- resulta vital para el funcionamiento de cualquier organización; si el elemento humano está dispuesto a proporcionar su esfuerzo, la organización marchará; en caso contrario, se detendrá.

Administrar una organización es llegar a los miembros de la misma hacia el logro de las metas establecidas aprovechando sus destrezas y habilidades para alcanzar la eficiencia del grupo y los resultados óptimos (Alles 2016).Por ello, para crear una organización educativa de calidad y competitiva se requiere la creación de las condiciones necesarias para ello, esto es, de medios y materiales, de gestión del 
conocimiento y de desarrollo del talento humano para lograr los objetivos misionales (Núñez 2017).

A guisa de conclusión final, se puede afirmar que las competencias administrativas de los gestores educativos es una de las variables de gestión educativa que se torna muy importante y determinante para el logro de la calidad educativa.

\section{Referencias}

Alles, M. A. (2016). Selección por competencias. Buenos Aires: Ediciones Granica SA.

Ayala, J. (2005). Programa de Certificación de Competencias Laborales. Competencias Básicas en Gestión., Buenos Aires: Banco Interamericano de Desarrollo.

Beresauluce, M. (2008). La calidad como reto en las escuelas de educación infantil al inicio del siglo XXI: Las escuelas de Reggio Emilia, de Loris Malaguzzi, como modelo a seguir en la práctica educativa. (Tesis Doctoral). Universidad de Alicante. En http://hdl.handle.net/10045/11273

Blanco, M. (2005) La educación de calidad para todos empieza en la primera infancia. $\begin{array}{lllll}\text { Revista enfoques educacionales } & 7 & \text { (1), } & 11-33 & \text { En }\end{array}$ http://www.facso.uchile.cl/publicaciones/enfoques/09/BlancoDelPiano_N7_2005.pdf

Bolívar, A. (2009). Una dirección para el aprendizaje. Revista electrónica sobre Calidad, Eficacia y Cambio en Educación, 7 (1), 1-4.

Cano, E. (1998). Evaluación de la calidad educativa. Madrid: La Muralla. URl: http://terras.edu.ar/biblioteca/12/ECPI_Cano_3_Unidad_1.pdf

Castro, A. (2015) Competencias académicas y administrativas en el profesorado de educación media superior. Revista Mexicana de Investigación Educativa, 20 (64), 263-294. http://www.comie.org.mx/documentos/rmie/v20/n064/pdf/64012.pdf

Chiavenato, I. (2005). Introducción a la teoría general de la administración. México: McGraw Hill Interamericana.

Contreras, T. (2016). Liderazgo pedagógico, liderazgo docente y su papel en la mejora de la escuela: una aproximación teórica. Propósitos y Representaciones, 4 (2), 231-284. Doi: http://dx.doi.org/10.20511/ pyr2016.v4n2.122.

Cossio, J. (2014). Pedagogía y calidad de la educación: una mirada a la formación del $\begin{array}{lllll}\text { maestro } & \text { rural. } & \text { Sophia, } & 10 & \text { (1), } \\ 14-23 . & \text { En }\end{array}$ http://www.scielo.org.co/pdf/sph/v10n1/v10n1a02.pdf

Del Arco, M. J. (2007). Los directivos de los centros escolares en el sistema educativo. CEE Participación Educativa, 5.

Dextre, J. y Del Pozo, R. (2012). ¿Control de gestión o gestión del control? Revista Contabilidad y Negocios, 7 (14), 69-80. En http://www.redalyc.org/pdf/2816/281624914005.pdf

Díaz, J., Núñez, L y Cáceres, K. (2018) Influencia de las competencias gerenciales y la gestión por resultados en la imagen institucional. Revista Fides et Ratio, 16, 169 -197. 
En http://www.ulasalle.edu.bo/fidesetratio/index.php/4-fides-et-ratio-volumen-16/110-fides-et-ratio-volumen-16-completo\#revista.

Escudero, J., Delfín, L. y Arano, R. (2014) El desarrollo organizacional y la resistencia al cambio en las organizaciones. Ciencia administrativa, 1, En https://www.uv.mx/iiesca/files/2014/09/portada201401.pdf

Galán. A. (2010). Justificación y Limitaciones de la Investigación. En http://google.academico.com/2010/02/justificacion-y-limitaciones-en-la.html.

Gallego, J. L. y Rodríguez, A. (2014). El reto de una educación de calidad en la escuela inclusiva. Revista Portuguesa de Pedagogía, 48 (1), 1-24. En http://impactumjournals.uc.pt/index.php/rppedagogia/article/view/2237/1476

Gonzales, A. (2004). Evaluación del clima escolar como factor de calidad. Madrid: La Muralla. ISBN 13: 9788471337429.

Hernández, R., Fernández, C., y Baptista, P. (2014). Metodología de la investigación. Sexta Edición. México D.F.: McGraw-Hill.

Hernández, R., Fernández, C., y Baptista, P. (2014). Metodología de la investigación. Sexta Edición. México D.F.: McGraw-Hill.

Lorenzo, M. (2012). Las comunidades de liderazgo de centros educativos. Educar, 48 (1) , 9-21. https://www.raco.cat/index.php/Educar/article/view/252990

Majad, M. (2016). Gestión del talento humano en organizaciones educativas. Revista de investigaciones, 88 (40).

Medina, M., Armenteros, M, Guerrero, L. y Barquero, J. (2012). Las competencias gerenciales desde una visión estratégica de las organizaciones: un procedimiento para su identificación y evaluación del desempeño. Revista Internacional Administracion \& Finanzas, 5 (2), En ftp://ftp.repec.org/opt/ReDIF/RePEc/ibf/riafin/riaf-v5n22012/RIAF-V5N2-2012-6.pdf

Mejía-Giraldo, A., Bravo-Castillo, M. y Montoya-Serrano, A. (2013). El factor del talento humano en las organizaciones. Revista Ingeniería Industrial, 34 (1). En http://scielo.sld.cu/scielo.php?script=sci arttext\&pid=S1815-59362013000100002

Miranda, J. (2011). Reflexiones sobre la calidad de la educación y sus referentes: el caso de México. Revista Venezolana de Educación Educere.

Murillo, Z. (2011). Enfoques cuantitativo y cualitativo de la investigación en ciencias sociales. En

http://www.tlalpan.uvmnet.edu/oiid/download/Enfoques\%20cualitativo\%20cuan titativo_04_CSO_PSIC_PICS_E.pdf

Núñez, L. (2017). Sistema de gestión de la calidad en educación. Lima: Ediciones Multigrafik.

Núñez, L., Bravo, L., Cruz, C. e Hinostroza, M. (2018). Competencias gerenciales y competencias profesionales en la gestión presupuestaria. Revista Venezolana de Gerencia, 23 (83).

Paladines. L. (2012). El Proceso de Mercadotecnia y la Gestión de Comercialización de Bienes y Servicios. Universidad Nacional de Loja Área Jurídica. Social y Administrativa.

Rodríguez, L. (2014). Estudio de los Espacios Arquitectónicos-Educativos en la 
Formación Profesiona. (Tesis Doctoral). Universidad de Granada

Rosselló, M. y Pinya, C. (2014). La formación en competencias básicas: un reto para la administración. Revista Profesorado,

18

(2).

En http://www.ugr.es/ recfpro/rev182COL6.pdf

Salazar, D. y Romero, G. (2006). Planificación. ¿Éxito Gerencial? Multiciencias, 6 (1). En http://www.redalyc.org/pdf/904/90460103.pdf

Unesco (2007). Educación de calidad para todos, un asunto de derechos humanos. Documento de discusión sobre políticas educativas en el marco de la II Reunión Intergubernamental del Proyecto Regional de Educación para América Latina y el Caribe (EPT/PRELAC). Buenos Aires, Argentina. En http://unesdoc.unesco.org/images/0015/001502/150272s.pdf

Vera. A. (2008). Tipos de Investigación. Bogotá: Colombia. Mc Graw - Hill.

Zorrilla. A. (1993). Principales tipos de investigación. URl: http://www.unac.edu.pe/documentos/organizacion/vri/cdcitra/Informes Finales Investigacion/IF_ABRIL_2012/IF_ALFARO\%20RODRIGUEZ_FIEE.pdf 